\title{
METODOLOGÍA MEDHIVE: DESARROLLO DE COMPETENCIAS DIGITALES EN EL CONTEXTO EDUCATIVO
}

\section{MEDHIVE METHODOLOGY: DEVELOPMENT OF DIGITAL COMPETENCES IN THE EDUCATIONAL CONTEXT}

\author{
DOI: www.doi.org/10.54198/innova08.08 \\ (iD) Américo Sirvente ${ }^{l}$ \\ Tamara Abigail Bitar ${ }^{2}$ \\ D) Iris Jiménez Pitre ${ }^{3}$
}

Cómo citar esté artículo: Sirvente, A., Bitar T., \& Jiménez, I., (2021). Metodología MeDHiVE: desarrollo de competencias digitales en el contexto educativo, Revista Innova ITFIP, 8 (1), 98-105

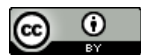

Recibido: Enero de 2021 Aprobado: Mayo de 2021

\section{Resumen}

El presente artículo tiene por objeto determinar la relación entre la planeación financiera y rentabilidad en empresas prestadoras de servicios de bombeo electro sumergible en el campo Moporo de PDVSA, estado Zulia, Venezuela. Para ello, se llevó a cabo una investigación descriptiva, correlacional con diseño no experimental de campo y transaccional, apoyada en la encuesta como instrumento la cual fue aplicada a diferentes personas en el área financiera y gerencial de las empresas Baker Hughes a GE Company y Schlumberger. La investigación arrojó como resultado que las empresas mencionadas anteriormente consideran el presupuesto de ingresos y egresos en su planificación, sin embargo, tienen problemas en considerar

\footnotetext{
1 Ingeniero. Director del Centro Tecnológico Universidad de San Juan Argentina Correo-e: americosirvente@gmail.com +542644696496, Orcid: https://orcid.org/0000-0001-7931-312X

${ }^{2}$ Doctora Directora Proyecto Universidad de San Juan Argentina, Docente investigador, Correo-e: tamaraabigailbitar@gmail.com +542644176593, Orcid: https://orcid.org/0000-0002-7262-8740

${ }^{3}$ Doctora. Docente investigadora, Universidad de la Guajira Colombia. Correo-e: iajimenez@uniguajira.edu.co +573008376440 , Orcid: https://orcid.org/0000-0002-8109-7013
} 
el presupuesto de efectivo al no poder controlar la cantidad que requieren para afrontar las necesidades ordinarias de recursos y no poder incluir las entradas esperadas de efectivo para el periodo financiero especifico, además se concluye que efectivamente las empresas consideran el presupuesto de capital y presupuesto de utilidades para elaborar los planes financieros a largo plazo, del mismo modo se observa que el margen de utilidades y la rotación de activos son calculados por las empresas para el cálculo de la rentabilidad económica, del mismo modo tanto el margen de utilidades como la rotación de activos son estudiados para determinar la rentabilidad financiera. Finalmente se obtuvo que la planeación financiera y la rentabilidad tienen una relación débil en el presente estudio con un valore de correlación de Pearson de 0,36.

Palabras clave: Competencias digitales, Tic, Tecnologías en entornos educativos, YouTube

\begin{abstract}
:
The purpose of this article is to determine the relationship between financial planning and profitability in companies providing electro-submersible pumping services in pdvsa's Moporo Field, Zulia state, Venezuela. To this end, a descriptive, correlational research was carried out with non-experimental field and transactional design, supported by the survey as an instrument which was applied to different people in the financial and managerial area of the baker hughes companies to GE Company and Schlumberger. The research showed that the companies mentioned above consider the revenue and expenditure budget in their planning, however, they have problems considering the cash budget by not being able to control the amount they require to meet the ordinary needs of resources and not being able to include the expected cash inflows for the specific financial period, in addition it is concluded that companies do indeed consider the capital budget and profit budget to prepare long-term financial plans, in the same way it is observed that the profit margin and the rotation of assets are calculated by the companies for the calculation of the economic profitability, in the same way both the profit margin and the turnover of assets are studied to determine the financial profitability. Finally, it was obtained that financial planning and profitability have a weak relationship in the present study with a Pearson correlation value of 0.36 .
\end{abstract}

Keywords: Digital skills, ICT, Technologies in educational environments, YouTube

\title{
Introducción
}

La forzada y repentina necesidad de usar herramientas digitales en la educación, hizo más evidente, una realidad que algunos investigadores intuían. Luego de que Marc Prenzky, 2001 definiera los "Nativos e Inmigrantes digitales", dando por sentado que las diferencias entre Nativos Digitales e Inmigrantes Digitales son: 
- Quieren recibir la información de forma ágil e inmediata.

- Se sienten atraídos por multitareas y procesos paralelos.

- Prefieren los gráficos a los textos.

- Se inclinan por los accesos al azar (desde hipertextos).

- Funcionan mejor y rinden más cuando trabajan en Red.

- Tienen la conciencia de que van progresando, lo cual les reporta satisfacción y recompensa inmediatas.

- Prefieren instruirse de forma lúdica a embarcarse en el rigor del trabajo tradicional.

Algunas de las aseveraciones planteadas por el autor antes citado, dependen mucho del contexto, la situación social, el nivel de integración tecnológica que recibió en su formación.

Antes de la cuarentena, el problema radicaba, en que las personas no podían aprender tan rápido el uso de las nuevas tecnologías, por lo que muchos mantenían un conocimiento obsoleto. Y en algunas ocasiones presentaban resistencia al cambio. En la actualidad, se atraviesa por una situación mundial debido a la pandemia, este agente de la naturaleza obligó a la humanidad a actualizarse o a quedar relegado, entre ellos, los alumnos. Diversas publicaciones indican que la imposición del aislamiento y/o confinamiento, ha potenciado el uso de la tecnología para el teletrabajo, las actividades bancarias y fundamentalmente la educación, aseverando que en este escaso tiempo, hemos avanzado lo que en épocas normales hubiésemos alcanzado en seis años.

Asimismo, cuando pensamos en Resiliencia reflexionamos conjuntamente acerca de las posibilidades de sobreponernos a estas y otras adversidades de manera positiva y constructiva; conscientes de que la solidaridad y participación grupal y comunitaria contribuirán a un mejor vivir, los ambientes académicos, en este contexto no acotados simplemente al aula física, constituyen los espacios simbólicos a través de los cuales transitamos estas nuevas y desconocidas cotidianeidades que demandan a su vez innovadoras experticias y destrezas singulares.

La investigación, llevada a cabo en la Facultad de Ciencias Sociales, con estudiantes de los últimos años de la carrera Licenciatura en Trabajo Social, más específicamente en la Cátedra Orientación Salud, el Proyecto denominado "TECNOLOGÍAS DE LA INFORMACIÓN Y COMUNICACIÓN Y RESILIENCIA EDUCACIONAL: Nuevas didácticas para nuevos escenarios", se encuentra en su primer año de ejecución y no obstante ello presenta conclusiones bastante novedosas. Dicho Proyecto, aprobado y financiado por la Universidad Nacional de San Juan Argentina.

Con la implementación de esta Metodología de Diseño Hipermedia de Videos Educativos (MeDHiVE) se desarrollaron más de 450 objetos virtuales de aprendizaje (OVAs) bien formados (páginas web educativas) y más de 2500 docentes adquirieron competencias digitales en los 
talleres presenciales impartidos, los OVA disponibles en un repositorio virtual (portalhuarpe.com.ar). MeDHiVE surge como una necesidad ante la aparición de la pandemia y permite ordenar la documentación referida al diseño, uso de recursos y secuencia pedagógica de un video educativo, disponible en la plataforma YOUTUBE.

Objetivos: Esta investigación se planteó como objetivo principal conocer nuevas tecnologías de información y comunicación desde el Principio Aprender a Hacer (UNESCO) y la Resiliencia Educacional con la puesta a prueba de YouTube concebida como herramienta didáctica académica en la educación superior.

\section{Materiales y métodos:}

Se optó por aplicar el tipo de metodología Acción Participantes, apoyado bajo la utilización de instrumentos de recolección de información plasmados a través de encuestas, cuestionarios, además se realizó un proceso de observación permanente para evaluar el avance en el desarrollo de las competencias digitales del estudiantado. Se aplicó metodología de diseño hipermedia de Videos Educativos (MeDHiVE) que es una adaptación funcional de la Metodología de Diseño hipermedial de Materiales Educativos (MeDHiME), de amplio éxito, con la que se desarrollaron más de 450 objetos virtuales de aprendizaje (OVA) bien formados (páginas web educativas) y más de 2500 docentes adquirieron competencias digitales en los talleres presenciales impartidos, los OVA disponibles en un repositorio virtual (portalhuarpe.com.ar)

\section{Resultados y Discusión}

Los resultados esperados que oportunamente esbozamos en el diseño de investigación fueron:

- Que se promueva sistemáticamente la Resiliencia Educacional con la implementación de tecnologías innovadoras basadas en principios establecidos por la UNESCO (Aprender a Hacer).

- Que los/as estudiantes, docentes e investigadores/as involucrados logren una integración positiva y sinérgica a lo largo de los procesos de aprendizaje

- Que una vez visualizados y registrados los principales hallazgos puedan formularse nuevas líneas de investigación-acción participante

- Que los resultados positivos de estas nuevas estrategias/prácticas pedagógicas puedan ser replicadas por otros/as docentes y estudiantes en otros contextos.

Usamos la Metodología de Diseño Hipermedia de Videos Educativos (MeDHiVE) que es una adaptación funcional de la Metodología de Diseño Hipermedial de Materiales Educativos (MeDHiME), de amplio éxito, con la que se desarrollaron más de 450 objetos virtuales de aprendizaje (OVA) bien formados (páginas web educativas) y más de 2500 docentes adquirieron 
competencias digitales en los talleres presenciales impartidos, los OVA disponibles en un repositorio virtual (portalhuarpe.com.ar).

MeDHiVE surge como una necesidad ante la aparición de la pandemia y permite ordenar la documentación referida al diseño, uso de recursos y secuencia pedagógica de un video educativo, disponible en la plataforma YOUTUBE.

Tiene tres etapas, que se plasman en una plantilla electrónica.

- La primera es el Análisis del DOMINIO, donde se define el título, objetivos, autores, palabras claves y otros elementos necesarios para definir el QUE se pretende hacer.

- $\quad$ La segunda se refiere a los RECURSOS digitales necesarios para el video, donde se dan pautas de búsqueda, selección y recuperación de los objetos digitales que pueden ser parte del video, o sea definir CON QUE haremos el video.

- La tercera documenta la SECUENCIA PEDAGÓGICA, de estos recursos, para constituir una unidad de enseñanza, lo que significa el COMO mostrarlos.

En toda la formación se instruye sobre uso del humor, herramientas de edición, tip de diseño, herramientas de evaluación y seguimiento para lograr un video atractivo que atrape a la audiencia.

En cuanto al avance concreto del estudio, a la fecha hemos realizado una planificación de clases, enfocadas al segundo semestre que incluyeron:

1. Metodología de Clase Inversa, donde se exponen las temáticas en una plataforma con los recursos digitales, para que los alumnos la consulten ANTES de la clase por videoconferencia.

2. Especificaciones conceptuales atinentes a los temas del programa de la Cátedra.

3. Entrega de material virtual (plano, en su mayoría en formato PDF) para estudio y elaboración de nuevas herramientas de estudio.

4. Uso de la plataforma CLASSROOM de Google, para la planificación y acceso a los materiales.

5. Generación de grupos de 3 y 4 alumnos para el trabajo colaborativo en el diseño y creación del video educativo global.

6. Utilización de DRIVE para el almacenamiento de documentos y trabajo colaborativo. 
7. Videoconferencias semanales, con Zoom, Jitsi o Meet, según estado de las redes.

8. Utilización de canal de YouTube, disponiendo de un conjunto de videos instructivos desarrollados por la cátedra.

9. Autoevaluación mediante Edpuzzle, que permite realizar preguntas dentro del video y determinar el nivel de apropiación de cada estudiante.

10. Comunicación mediante la plataforma y WhatsApp, en una canal general para todos las/os estudiantes y canales por grupo, para las devoluciones particulares.

11. Clases de consultas previamente pautadas para el tratamiento y colaboración en temas específicos.

En este momento hemos realizado el $90 \%$ de las actividades planificadas para esta primera etapa de acuerdo con nuestro cronograma de equipo, y se cuentan con la totalidad de los videos desarrollados por cada equipo de alumnos.

Las conclusiones preliminares, basadas en las observaciones, nos indican que hasta el momento los supuestos planteados por los autores del Proyecto se cumplen en un $75 \%$ aproximadamente (estando actualmente en un período de análisis y construcción de datos). Los mismos, oportunamente detallados en el diseño de investigación, se detallan también a continuación:

A) El manejo de nuevas TIC contribuirán a la democratización de la Prácticas/Estrategias expresadas desde las dimensiones del Principio Aprender a Hacer (UNESCO)

B) La implementación de una experiencia con una didáctica innovadora conllevará un salto cualitativo en las prácticas pedagógicas traducido en estrategias académicas Resilientes/Saludables

C) La utilización de tecnologías innovadoras (virtuales y reales) favorecerán la generación de Espacios Resilientes

Sin embargo, aparecen en el estudio algunas situaciones nuevas (o resultados no esperados) que se consideran igualmente valiosos en cuanto a riqueza epistemológica, por lo tanto, se las incorpora al análisis general; las mismas pueden ser expresadas de la siguiente manera:

1) No todos las/os se mostraban impacientes por recibir la información en forma inmediata, una porción minoritaria no consultaba el material propuesto.

2) Les resultaba dificultoso realizar actividades multitareas en sus computadores, por ejemplo, abrir otra aplicación en paralelo a la videoconferencia.

3) También surgieron problemas en el uso de herramientas dentro del trabajo en red. En nuestra estimación, suponíamos que sabían almacenar y usar recursos en la nube, pero en la realidad revistió cierta dificultad que realizaran documentos compartidos. 
4) En las actividades de autoevaluación, supuso problemas el cumplimiento de los trabajos en tiempo y forma; posiblemente por lo novedoso del sistema, fuera de lo habitual (en especial en tareas tales como responder preguntas a medida que visualizaban los videos).

\section{Conclusiones}

Esta primera evaluación, las cuales estamos en instancias de valorar y validar con mediciones más rigurosas, hemos notado que las/os nativas/os digitales, si bien poseen habilidades y competencias para algunas actividades de comunicación, no tienen las necesarias para la búsqueda de recursos educativos, uso de la nube, la búsqueda de contenido apropiado para su instrucción, entre otros.

Para subsanar este inconveniente durante la marcha, hemos reforzado el apoyo virtual en el canal de cada grupo, clases adicionales de consultas, entre otras actividades.

Hacia el futuro, cuando se aplique este diseño a nuevas poblaciones estudiantiles de similares características se considerará primordialmente la puesta en marcha de elementos que operen como niveladores conceptuales en cuanto al uso de las TIC para facilitar el posterior proceso. Asimismo, y como parte de una concepción de docencia compartida, se prevé la utilización del material didáctico co-elaborado con estudiantes en esta primera etapa, como insumo fundamental para construir nuevos recursos pedagógicos.

\section{Recomendaciones}

Este proyecto plantea en el segundo año, la evaluación de los productos tecnológicos generados a través de la metodología MeDHiVE, mediante la herramienta EDPUZZLE para lograr un producto final de alto nivel.

\section{Referencias bibliográficas}

Sirvente, A.-2006. Docentes no informáticos, materiales en Internet y MeDHiME (Metodología de Diseño Hipermedial de Materiales Educativos) -Capítulo 12-pág. 135-143 libro “CLAVES Y RETOS de las TIC's en la formación de Recursos Humanos-Propuestas, investigaciones, reflexiones y casos-EDUTIC 2006-Buenos Aires

Torres, E y Reus, S. (2005). Determinación De Variables con vistas a evaluar MEDHIME. III Congreso Internacional de Matemática Aplicada a la Ingeniería y Enseñanza de la Matemática en Ingeniería. Facultad de Ingeniería. Universidad de Buenos Aires. 2005.

Rivero, F. -2011-EL USO DEL HUMOR EN LA ENSEÑANZA: UNA VISIÓN DEL PROFESORADO DE ELE-Tesis de Maestría-Universidad de Jaén-España 
González Barrientos, C. -2013. El Humor como instrumento pedagógico. Tesis de LicenciaturaUniversidad de Chile

JESÚS DAMIÁN FERNÁNDEZ SOLÍS-EL VALOR PEDAGÓGICO DEL HUMOR. DOSSIER N. ${ }^{\circ}$ 44-REVISTA TRIMESTRAL DE LA ASOCIACIÓN PROYECTO HOMBRE

Sirvente, FA, (2007) "MeDHiME-Materiales Educativos Navegables-una metodología fácil para introducirá los docentes no informáticos en la web"-publicado por la Editorial de la Fundación de la Universidad Nacional de San Juan-ISBN 978-950-605-504-2. Sirvente, FA (2012) “Lifting Académico-Tus clases fácil y rápido en Internet con MeDHiME 2.0”publicado por la Editorial de la Fundación de la Universidad Nacional de San Juan-ISBN 978-950-605-709-1.

Ciancio, MI-Oliva, E. (2012) "Objeto de aprendizaje: Herramienta de la web 2.0 para álgebra lineal-Recurso didáctico exitoso" Editorial Académica Española (04.04.2012)-ISBN-13: 978-3-8484-7242-0, ISBN-10:3848472422, EAN: 9783848472420

Marc Prensky, 2001, "Digital Natives, Digital Immigrants", On the Horizon (MCB University Press, Vol. 9 No. 5, October 2001) y https://www.marcprensky.com/writing/PrenskyNATIVOS\%20E\%20INMIGRANTES\%20DIGITALES\%20(SEK).pdf 